\title{
Information-Driven Persistent Sensing of a Non-cooperative Mobile Target Using UAVs
}

\author{
Hyo-Sang Shin · Adrian Jesus Garcia · Sergio \\ Alvarez
}

Received: date / Accepted: date

\begin{abstract}
This paper addresses the persistent sensing problem of moving ground targets of interest using a group of fixed wing UAVs. Especially, we aim to overcome the challenge of physical obscuration in complex mission environments. To this end, the persistent sensing problem is formulated under an optimal control framework, i.e. deploying and managing UAVs in a way maximising the visibility to the non-cooperative target.The main issue with such a persistent sensing problem is that it generally requires the knowledge of future target positions, which is uncertain. To mitigate this issue, a probabilistic map of the future target position is widely utilised. However, most of the probabilistic models use only limited information of the target. This paper proposes an innovative framework that can make the best use of all available information, not only limited information. For the validation of the feasibility, the performance of the proposed framework is tested in a Manhattan-type controlled urban environment. All the simulation tests use the same framework proposed, but utilise different level of information. The simulation results confirm that the performance of the persistent sensing significantly improves, up to $30 \%$, when incorporating all available target information.
\end{abstract}

Keywords persistent sensing $\cdot$ unmanned aerial vehicles $\cdot$ behaviour recognition $\cdot$ path planning $\cdot$ information-driven tracking $\cdot$ target tracking

\author{
Hyo-Sang Shin \\ Cranfield University, College Rd, Cranfield, Bedford MK43 0AL, UK \\ Tel.: +44 (0) 1234758577 \\ E-mail: h.shin@cranfield.ac.uk \\ Adrian Jesus Garcia \\ Universidad Politecnica de Madrid. ETSI Caminos. Lab. Topografia y Geomatica, Madrid, 28040 Madrid, \\ Spain \\ Tel.: +34 913366670 \\ E-mail: adrian.garcia.sanchez.aero@gmail.com \\ Sergio Alvarez \\ Universidad Politecnica de Madrid. ETSI Caminos. Lab. Topografia y Geomatica, Madrid, 28040 Madrid, \\ Spain \\ E-mail: sergio.alvarez@upm.es
}




\section{Introduction}

The large scale of UAS applications has proliferated vastly within the last few years. The operational experience of UAVs has proven that their technology can bring a dramatic impact to military and civilian areas. This includes, but not limited to: obtaining real-time, relevant situational awareness; helping commanders to lead appropriate decision making; and reducing risk to the mission and operation. Especially, groups of multiple UAVs are of special interest due to their ability to coordinate simultaneous coverage of large areas, or co-operate to achieve common goals. Specific applications under consideration include border patrol [1-3], airborne surveillance [4-6], police law enforcement [7,8] and forest-fire localization [9]. One of the primary capabilities of UAVs to enable these applications is searching and subsequent tracking of moving ground targets of interest.

It has been shown that the effectiveness of these capabilities can be maximised in complex environments such as an urban environment. However, these environments also raise serious challenges in operating UAVs. Especially, buildings and structures generate physical obscuration and occlusion of the line of sight (LOS) which can be the detriment of the operational capabilities of UAVs.

The main motivation of this paper is to address the challenge of physical obscuration in the persistent target tracking problem for a group of fixed wing UAVs. In particular, this paper aims for smart ways of deploying and managing UAVs in the urban environment to mitigate the effects of physical obscuration on persistent sensing for a non-cooperative target.

This paper develops a persistent sensing algorithm under an optimal control framework. The proposed algorithm aims to design trajectories of UAVs to maximise the visibility of the target in consideration of the physical obscuration, while meeting other objectives. The visibility to be maximised includes the LOS availability to the current target position, as well as to its future positions. The prediction inherently contains uncertainties, since future positions of a non-cooperative target can be only guessed. Therefore, this paper adopts a probabilistic approach which finds the probability distribution of the future target positions and gives a priority to the maximisation of LOS availability to the more probable future positions of the target.

The key innovation of this paper is to make the best use of available information in obtaining the probability distribution of the future target positions. Typically, only the track information and dynamics of the target are utilised in calculating the probability distribution of the predicted target positions. There is obviously certain information correlated with the future target positions, for example, contextual information including the given environment such as road network and the target's intent. The target is most likely to move along the road, so the probability distribution of the future target positions should be mainly propagated along the road. When the target has intent, that will influence its future positions. The probability of the predicted target position should increase if the future target position complies with a specific behavioural pattern identified. Leveraging this information will dramatically reduce uncertainties on the probability distribution of the future target positions and, consequently, improving the performance of the persistent sensing.

There have been extensive researches on behaviour monitoring of ground targets which endeavour to identify the target's intent and take proactive measures [10-12]. Thanks to the results of these studies, it is possible to utilise the behavioural patterns and intents of the target in persistent sensing. However, to the best of the authors' knowledge, there is no persistent sensing/tracking approach that incorporates the behavioural patterns of the target identified. 
Therefore, this paper first designs a framework enabling utilisation of the target's behavioural information in obtaining the probability distribution of the future target positions. Then, we design a simple persistent sensing guidance algorithm. Next, this paper validates the proposed approach, focusing on the investigation on how the performance changes as more available information is utilised. The proposed framework and validation stay at the level of feasibility study since the focus of this paper is to examine the proposed idea, that is using the information in the persistent sensing. Refinement and elaboration of the proposed approach will be subject of future studies. Note that the key contribution of this paper is complementary to other types of persistent guidance algorithms, i.e. the main idea of utilising all available information can be easily incorporated into other persistent guidance algorithms.

\section{Previous Works}

Circular flights are recommended for various target tracking applications as for each UAV the maximum altitude flight ensures the maximum visibility and the minimum radius turn keeps the minimum distance to the target at the maximum altitude [13]. A common approach which follows these principles is the standoff LOS tracking concept. In this approach, the UAV closely orbit around the target maintaining sensor coverage and remaining outside a critical threat range for the persistent tracking. In [14-17], vector field-based approaches were proposed to guide the UAV to a stable vector field around a target. Zhu et al. and Shames et al. $[18,19]$ addressed a tracking problem to make the UAV loiter around a target such that both the target estimator and the control systems are stable. Optimal path planning approach is also proposed to provide convoy overwatch for a moving ground vehicle in [20]. Rafi et al. [21] added the control of a gimbaled sensor to a circular loiter problem. Wise and Rysdyk [22] compared the different methodologies for circular LOS tracking. Standoff target tracking using cooperative UAVs is also proposed by distributing a team of UAVs on a standoff orbit when the target vehicle is uncooperative, or is highly agile [23-29]. However, most of these research on standoff tracking neglected the physical obscuration issues and it is generally difficult to properly handle the mobility of the target in maximising visibility under this type of tracking.

A few optimal frameworks have been also applied to the tracking problem for persistent sensing. A model predictive control (MPC) algorithm, was introduced by $\mathrm{He}$ and Xu [30] in order to obtain the optimal path fulfilling the dynamic constraints of the problem. Yao et al. [31] combined the MPC with an improved version of the Lyapunov Guidance Vector Field wchich introduces flight height and considers complex dynamic constraints. Game theory has also been successfully applied by Zhang and Liu [32] in order to address the persistent tracking problem by modelling the problem as a pursuit-evasion game. Wang and $\mathrm{Cao}$ [33] proposed a new framework technique based on chemical reaction optimization and considering flight dynamic and collision-free constraints . Tang and Ozguner [34] analysed the problem of multi target tracking proposing a gradient-approximation algorithm to travel through a series of defined target points achieving a cooperative tracking. However, no occlusions to the sensors due to the environment were considered.

Xung et al. [35] introduced a guidance law and heading rate control that allow the minimisation of the exposure time proving their approach in a simplified environment. Ramasamy and Ghose [36] proposed an algorithm that maximizes the visitation on preferential areas and that reduces the frequency of risk areas visiting using a heuristic learning technique. Redding et al. [37] approached the issue with an intelligent Cooperative Control 
Architecture implementing an active learning approach. Grocholsky et al. [38] proposed an information based approach to a cooperative air and ground tracking problem. Although this approach provides good results in case of tracking static objects, it is not suited when considering non-cooperative ground targets in urban environments. Shaferman and Shima [39] applied a Genetic Algorithm (GA) which generates paths maximising LOS availability in consideration of airspace limitations. GA are also used by Zhang et al. [40] for the optimal tunning of the control law.

A bayesian filtering method incorporated with a Hospitality map was proposed by Tang and Ozguner [41] in order to address the tracking of terrain-dependant behaviour ground targets. Partially Observable Markov Decision Processes (POMDPs) were proposed by Capitan et al. [42,43] although the implementation leads to a great computational complexity. Moreover, Ramasamy et al. [44] presented a heuristic learning algorithm which allows the definition of quantitative priorities per region.

Yu et al. [45] proposed a suboptimal probabilistic approach where the path is selected from a path planning tree after the optimisation of a cost function. The number of paths considered in this approach grows exponentially with the length of the look-ahead window. In order to avoid this issue, Cook et al. [46] considered the same amount of paths regardless of the length of the prediction horizon and optimally selected a path based on an auction scheme. These approaches assumed a prior distribution of the target future position as known. An equiprobability approach, using a normal distribution around the last known target's position was proposed by Bourgault et al. [47]. Kim and Kim [13] also proposed a tracking algorithm which aims to maximise the chance to keep the target inside the camera field of view. This algorithm used uniform random samples taken from the ground and obstacles in the region of interest and constructed a performance index using those samples.

It is evident that there have been extensive studies on the persistent sensing/tracking problems. However, the simulation results of these studies, with an average persistent sensing of around $60-80 \%$ in scenarios with one target and 2 tracking sensors, show that there is still a great margin of improvement in the persistent problem solution. Moreover, there is yet no persistent sensing/tracking approach utilising target's intents and/or behavioural patterns.

\section{Mathematical model}

\subsection{Initial assumptions}

For the simplicity, this paper only focuses on the persistent sensing problem in $2 \mathrm{D}$ space. It is assumed that the non-cooperative target is initially detected by other means. To emphasise the potential advantages of utilising environment information, the target is assumed to travel only on the road. Note that this assumption can be easily relaxed. It is also assumed that an estimation algorithm is applied, so the current target position can be estimated with a certain degree of uncertainty so long as the LOS is maintained. When the propagation of the future target position is performed, this paper assumes that the target could be at any point of the road width. The wind disturbance is not considered in this paper as it is not the main focus. When designing guidance laws, it is usually assumed that the control system of the UAV is well designed and its bandwidth is well separated from the guidance loop. This paper accepts this assumption and only considers the guidance problem based on the separation principle. In addition, it is assumed that the UAVs are operated at a certain altitude always greater than the maximum height of the polyhedrons that represent the urban model. 


\subsection{Environment model}

In this paper, it is assumed that the environment map is given in prior. For simplicity, we have created a Manhattan-type urban environment which is decomposed by cells. A Manhattantype urban environment map example is depicted in Fig. 1. As shown, the map is divided by $10 \times 10$ squares. Each square either contains a building or is empty. The buildings' height can also be set as desired. Note that the representation of the environment map can be easily updated if needed.

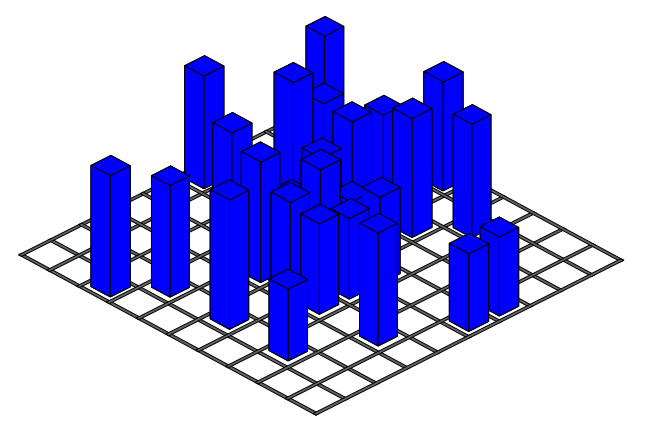

Fig. 1 Defined testing environment.

Let:

$$
\mathscr{B}=\{1,2, . . B\}
$$

be the set of buildings defined in the environment. Each building $B \in \mathscr{B}$ will be defined by a polyhedron's body, $\mathscr{E}_{B}$ characterized by the coordinates of its corners in the inertial frame and the height.

$$
\mathscr{E}_{B} \equiv z=H_{B}\{x, y\}, \quad\{x, y\} \in \boldsymbol{X}_{B}
$$

where $H_{B}$ is the height of building B and $\boldsymbol{X}_{B}$ is the region defined by the building's corners. Therefore, the urban environment, $\mathscr{E}$ will be given by the union of all the buildings:

$$
\mathscr{E}=\bigcup_{B=1}^{B=N_{B}} \mathscr{E}_{B}
$$

Now, let:

$$
\mathscr{M}=\left\{1,2, \ldots, N_{M}\right\}
$$

be the set of intersections in the road map. Each intersection $M \in \mathscr{M}$ will be defined by its coordinates in the inertial frame $\mathscr{I}_{M} \equiv\left(x_{m}, y_{m}\right)$. Therefore, all the intersections will be given by:

$$
\mathscr{I}=\bigcup_{M=1}^{M=N_{M}} I_{M}
$$


In addition, a direction has been assigned to the different roads. The directions can be single or double. The information of the directions of the roads will be stored in a field inside the intersection set, $\mathscr{I}$, which will contain a vector $\boldsymbol{d}_{M}$ containing the allowable directions. For example, if in the intersection $M$ the directions defined as allowable according to the traffic rules are north and east the vector would be: $\boldsymbol{d}=[1,1,0,0]$.

This paper naturally selects a cell decomposition to represent the environment map. Let $\mathscr{C}$ be the set given by:

$$
\mathscr{C}=\left\{1,2, \ldots N_{C}\right\}
$$

where $N_{C}$ is the number of cells in the map. Then, each cell, $\mathscr{R}_{C}$, is defined by the coordinates of its centre, $\left(\hat{x}_{c}, \hat{y}_{c}\right)$ and by a field that determines whether the cell is a road cell or not. The set of cells is then defined as $\mathscr{R}$ such that:

$$
\mathscr{R}=\bigcup_{C=1}^{C=N_{C}} \mathscr{R}_{C}
$$

\subsection{Mathematical model of the UAV kinematics and dynamics}

Under the assumptions given, we use UAV kinematics in 2D space, not full UAV dynamics. The geometry of the UAVs kinematics, modelled as discrete time dynamical systems, can be obtained as:

$$
\left\{\begin{array}{l}
\boldsymbol{x}_{i}(k+1)=\boldsymbol{f}\left(\boldsymbol{x}_{i}(k), \boldsymbol{u}_{i}(k)\right) \\
\boldsymbol{x}_{i}(k)=\left[x_{i}(k), y_{i}(k), \psi_{i}(k)\right]^{T}
\end{array} \quad \text {, for } i=\left\{1,2, \ldots, N_{U}\right\}\right.
$$

where $N_{U}$ is the number of UAVs, $x$ and $y$ denote the position in $x$ and $y$ axes, $\psi$ the heading angle, and $\boldsymbol{u}$ the commanded control input vector. The variables written in boldface imply that those variables are vectors and the functions in boldface represent vector valued functions. The time step is denoted as $k$ and the subscript $i$ on the variables indicates that those variables belong to the $i^{\text {th }}$ UAV. So, for example, $x_{i}(k)$ is the $x$ position of the $i^{\text {th }}$ UAV at time step $k$. Note that vectors are represented by the right-pointing arrow placed above their names in this paper.

Fig. 2 shows the geometry of UAV kinematics. In Fig. 2, the acceleration is denoted as $a$ and $v$ represents the velocity vector of the UAV. The continuous UAV's kinematics relative to the inertial frame is obtained as:

$$
\begin{aligned}
& \dot{x}_{i}(k)=v_{i} \cdot \sin \left(\psi_{i}(k)\right) \\
& \dot{y}_{i}(k)=v_{i} \cdot \cos \left(\psi_{i}(k)\right) \\
& \dot{\psi}_{i}(k)=\frac{a_{i}(k)}{v_{i}} \\
& u_{i}(k)=a_{i}(k)
\end{aligned}
$$

From simple discretisation of Eqn. (9), Eqn. (8) can be expressed as:

$$
\boldsymbol{x}_{i}(k+1)=\left[\begin{array}{l}
x_{i}(k+1) \\
y_{i}(k+1) \\
\psi_{i}(k+1)
\end{array}\right]=\left[\begin{array}{l}
x_{i}(k) \\
y_{i}(k) \\
\psi_{i}(k)
\end{array}\right]+\left[\begin{array}{c}
v_{i} \cdot \sin \left(\psi_{i}(k)\right) \\
v_{i} \cdot \cos \left(\psi_{i}(k)\right) \\
\frac{u_{i}(k)}{v_{i}}
\end{array}\right] \cdot \Delta T
$$

The motion of the UAV will be constrained by: 


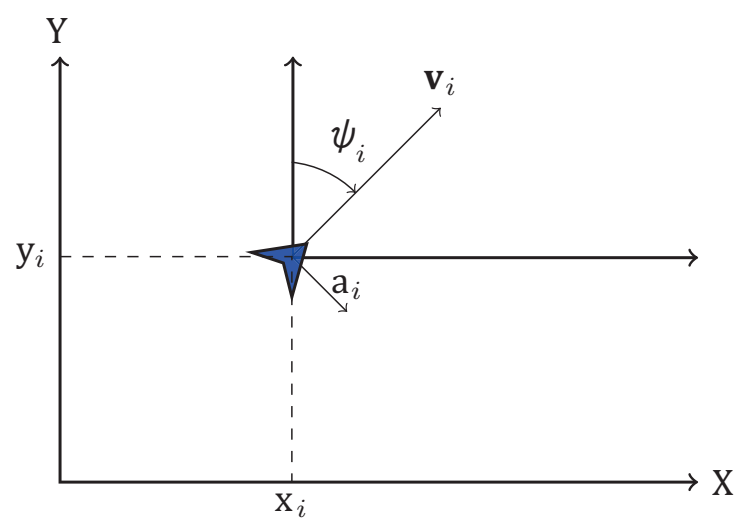

Fig. 2 Illustration of the UAV's kinematics.

- Minimum and maximum horizontal velocities. Thus, $v_{\min } \leq v_{i} \leq v_{\max }$.

- Minimum turning radius, $R_{\min }$, defined by the manoeuvrability of the platform.

- Maximum control input (commanded acceleration), $u_{\max }$ given by $u_{\max }=v^{2} / R_{\min }$

\subsection{Target model}

A schematic representation of the target's dynamics has been included in Fig. 3. The target will be modelled as a point mass. For simplicity, we assume that all the roads are at 0 altitude. However, this assumption can be readily removed. The state vector of the car at time step $\mathrm{k}$ will be defined by:

$$
\begin{aligned}
& \boldsymbol{x}_{T}(k)=\left[\boldsymbol{p}_{T}(k), \boldsymbol{v}_{T}(k)\right]^{T} \\
& \boldsymbol{p}_{T}(k)=\left(x_{T}(k), y_{T}(k), 0\right) \\
& \boldsymbol{v}_{T}(k)=\left(v_{T x}(k), v_{T y}(k), 0\right)
\end{aligned}
$$

where the subscript $T$ on variables is used to denote the variables of the target, $\boldsymbol{p}_{T}(k)$ and $\boldsymbol{v}_{T}(k)$ denote the position and velocity vectors of the target, respectively. On the other hand, the subscript $x$ and $y$ on variables represent projections of those variables onto the $\mathrm{x}$ and $\mathrm{y}$ axes, respectively.

The targets are not necessarily moving at a constant speed. The dynamics of the target considered is given by:

$$
\boldsymbol{x}_{T}(k+1)=\boldsymbol{x}_{T}(k)+\dot{\boldsymbol{x}}_{T} \Delta t
$$

where $\Delta t$ is sampling time.

The dynamics of the ground vehicle will be constrained by:

- A maximum turning velocity, $V_{1}$.

- A maximum velocity to perform a U-turn manoeuvre, $V_{2}$.

- A minimum turning radius, $R_{\min }^{T}$

In addition, it will be useful to define the course vector, $\boldsymbol{c}$ indicating in which direction the target is moving. The vector will contain the cardinal direction which will be defined by 


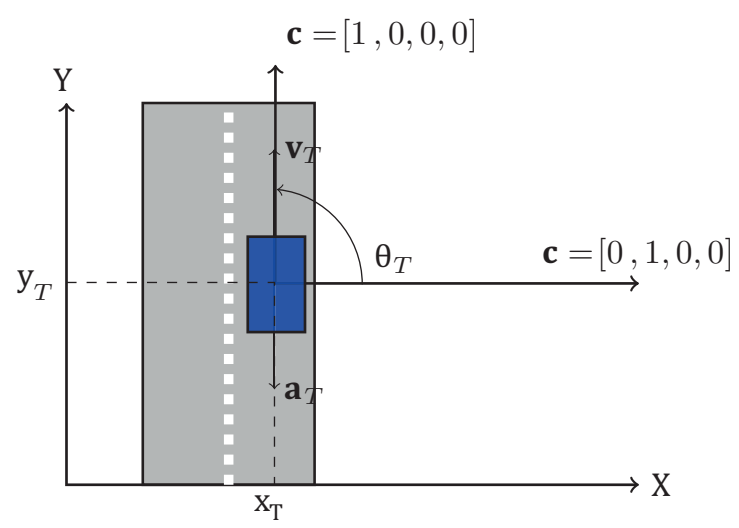

Fig. 3 Definition of the target dynamics: the course of the target is defined by the vector $c$

the velocity vector of the point of interest, $\boldsymbol{v}_{T}$,

$$
\theta_{T}(t)=\tan ^{-1} \frac{v_{T y}(t)}{v_{T x}(t)}
$$

Note that the angle $\theta_{T}(t)$ is the angle between the target's velocity vector and the $\mathrm{x}$ axis. Therefore:

$$
\begin{aligned}
& \theta_{T}=0 \rightarrow c=[0,1,0,0] \quad \text { East direction } \\
& \theta_{T}=\frac{\pi}{2} \rightarrow \boldsymbol{c}=[1,0,0,0] \quad \text { North direction } \\
& \theta_{T}=\pi \rightarrow c=[0,0,0,1] \quad \text { West direction } \\
& \theta_{T}=\frac{3 \pi}{2} \rightarrow \boldsymbol{c}=[0,0,1,0] \text { South direction }
\end{aligned}
$$

\section{Prediction of the future target positions}

Now, let us introduce how we obtain the probability distribution of the predicted future target positions.

To calculate the probability of future target positions, $N_{\text {sam }}$-number of samples are extracted using the available information such as dynamic model and behavioural patterns of the target and environment information. Then, based on the Euclidean distance between the position and centre of each cell, we determine to which cell the samples generated belong: each sample is assigned to the closest cell. Next, the probability associated to each cell, $\hat{\boldsymbol{x}}_{c}=\left(\hat{x}_{c}, \hat{y}_{c}\right)$, for a given time step, $t$, is computed as:

$$
P\left(\hat{\boldsymbol{x}}_{c}, t\right)=\frac{N_{\text {rep }}}{N_{\text {sam }}}
$$

where $N_{\text {rep }}$ is the number of samples, which are appeared in the cell, $\boldsymbol{x}_{c}$, as a possible future target position at the given time step $t$. 
If the target dynamics is not available, it is rational to assume that the target can be in any position within feasible region in the environment based on the position and velocity information of the target. The candidate region that includes the target can be given by:

$$
R_{f t p}:=\left\{\boldsymbol{p} \mid\left\|\boldsymbol{p}-\boldsymbol{p}_{T}(?)\right\| \leq r_{f t p} \text { and } \boldsymbol{p} \text { is not overlapped with any buildings }\right\}
$$

where $r_{f t g}$ is the radius of the candidate region and is given as

$$
r_{f t p}=v_{T, \max } \times t^{*}
$$

where $t^{*}$ denotes the look-ahead window, measured in time steps, for which the future target position will be predicted and $v_{T, \text { max }}$ represents the maximum speed of the target. If $v_{T, \max }$ cannot be estimated, we use the current speed of the target. From this candidate region, we generate $N_{\text {sam }}$ number of samples. The set of these samples can be written as:

$$
\left\{\begin{array}{l}
S_{f t p}:=\left\{S_{f t p, 1}, \ldots, S_{f t p, k^{*}}\right\} \\
S_{f t p, i}:=\left\{\hat{\boldsymbol{p}}_{T, t}(i, j) \text { for } j=1, \ldots, N_{s i m} \mid \hat{\boldsymbol{p}}_{T, t}(i, j)=r_{j}\left(\cos \theta_{j}, \sin \theta_{j}\right) \in R_{f t p}\right\}
\end{array}\right.
$$

where $\hat{\boldsymbol{p}}_{T, t}$ denotes the target position vector predicted at time step $t, N_{\text {sim }}$ represents the number of simulation and $k^{*}=t^{*} / \Delta t$. Here, $\Delta t$ is the sampling time and $N_{\text {sam }}=k^{*} \times N_{\text {sim }}$. Note that $r_{j}$ and $\theta_{j}$ are uniformly sampled as:

$$
\begin{aligned}
r_{j}^{2} & \sim U_{c}\left(0, r_{f t p}^{2}\right) \\
\theta_{j} & \sim U_{c}(0,2 \pi)
\end{aligned}
$$

where $U_{c}$ denotes the continuous uniform distribution. This sampling approach will generate random points uniformly distributed over the disk with the radius of $r_{f t p}$. Under the assumption that the environment information is available, all $\boldsymbol{p}_{T, t}$ are associated with the closest road cells.

If the target is lost or being lost, we use the same framework. However, in this case, $t$ in Eqn. (16) and $t^{*}$ in Eqn. (17) will be replaced by $k_{\text {lost }}$ and $\left(t+t^{*}-t_{\text {lost }}\right)$, respectively. Here, $t_{\text {lost }}$ denotes the time step when the target was lost.

When the information of the target dynamics is available, the target's position will be propagated through the target dynamics to generated samples. Since it is widely accepted that it is hard, if not impossible, to directly predict the target acceleration, we will predict the future target manoeuvres in terms of its turns and use them in the propagation. In this paper, the outcomes for the potential turn of the target are described by the sample space, $\Omega$, defined as:

$$
\Omega=\{\text { straight, right turn, left turn, u turn }\}
$$

We then define a random variable $(\mathrm{Rv}) X$ on this sample space as:

$$
X(\omega)= \begin{cases}0, & \text { for } \omega=\text { straight } \\ 1, & \text { for } \omega=\text { u turn } \\ 2, & \text { for } \omega=\text { right turn } \\ 3, & \text { for } \omega=\text { left turn }\end{cases}
$$

where $\omega$ represents a possible outcome in the sample space $\Omega$.

The probability mass function (PMF), $f_{X}(x)$, will be obtained using all the available information. If there is no information available, then it will be logical to assume that all the 
possible turn directions have the same probability. Hence, the PMF on the random variable is given by:

$$
f_{X}(x)=P(X=x)=U\{0,3\}
$$

where $U$ denotes the discrete uniform distribution. As it is assumed that the environment information is available, we use them to refine the PMF as:

$$
f_{X}(x)=E_{I} U\{0,3\}+\left(1-E_{I}\right) U\{0,1\}
$$

where $E_{I}$ is the index of the intersection that is given by:

$$
E_{I}= \begin{cases}0, & \text { if } \boldsymbol{p}_{T} \notin \mathscr{I}, \\ 1, & \text { if } \boldsymbol{p}_{T} \in \mathscr{I} .\end{cases}
$$

Eqn. (24) is based on the assumption that the target stays on the roads. This implies that all turn manoeuvres are possible at the intersections, whereas only moving straight and u-turn manoeuvre are possible on the other types of roads. The cumulative distribution function $(\mathrm{CDF})$ of the random variable $X, F_{X}(x)$, can be then constructed. If there is any other information, it will be utilised to refine the PMF. How to refine the PMF using available information will be discussed in following subsection in detail.

In order to determine the turn direction at each iteration of the prediction procedure, we generate a random number, $p_{s e l}$, from a continuous uniform distribution:

$$
p_{s e l} \sim U_{c}(0,1)
$$

The turn of the target at each iteration is elected based on $p_{\text {sel }}$ and the CDF. Once a turn direction is elected, the velocity components are updated accordingly. The procedure of determining the turn direction is described in Algorithm 1. The TURNELECTION returns the selected turn direction. Then, the velocity is updated using the elected turn direction and the future position of the target is predicted using the system equation defined in Eqn. (12) and updated velocity. The VELOCITYUPDATE, refer to Algorithm 2, details how to update the velocity based on the elected turn direction.

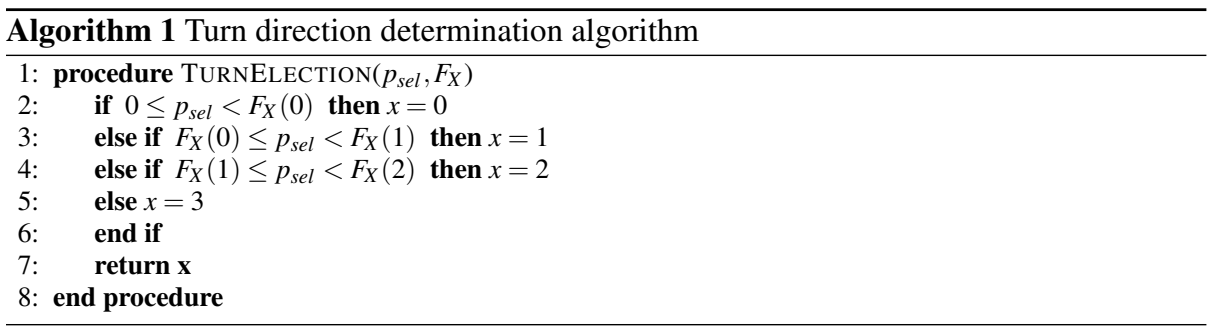

Algorithm 3 describes the main algorithmic procedure of obtaining the probability map of the future target positions, when the dynamics information of the target is available. In the algorithm, $A_{D I}$ and $A_{E P}$ denote availability of dynamics analysis and target's behavioural pattern information, respectively; 1 means available and 0 not available.

\subsection{Prediction Refinement}

Now, let us discuss how to refine the PMF if there is additional information available. 

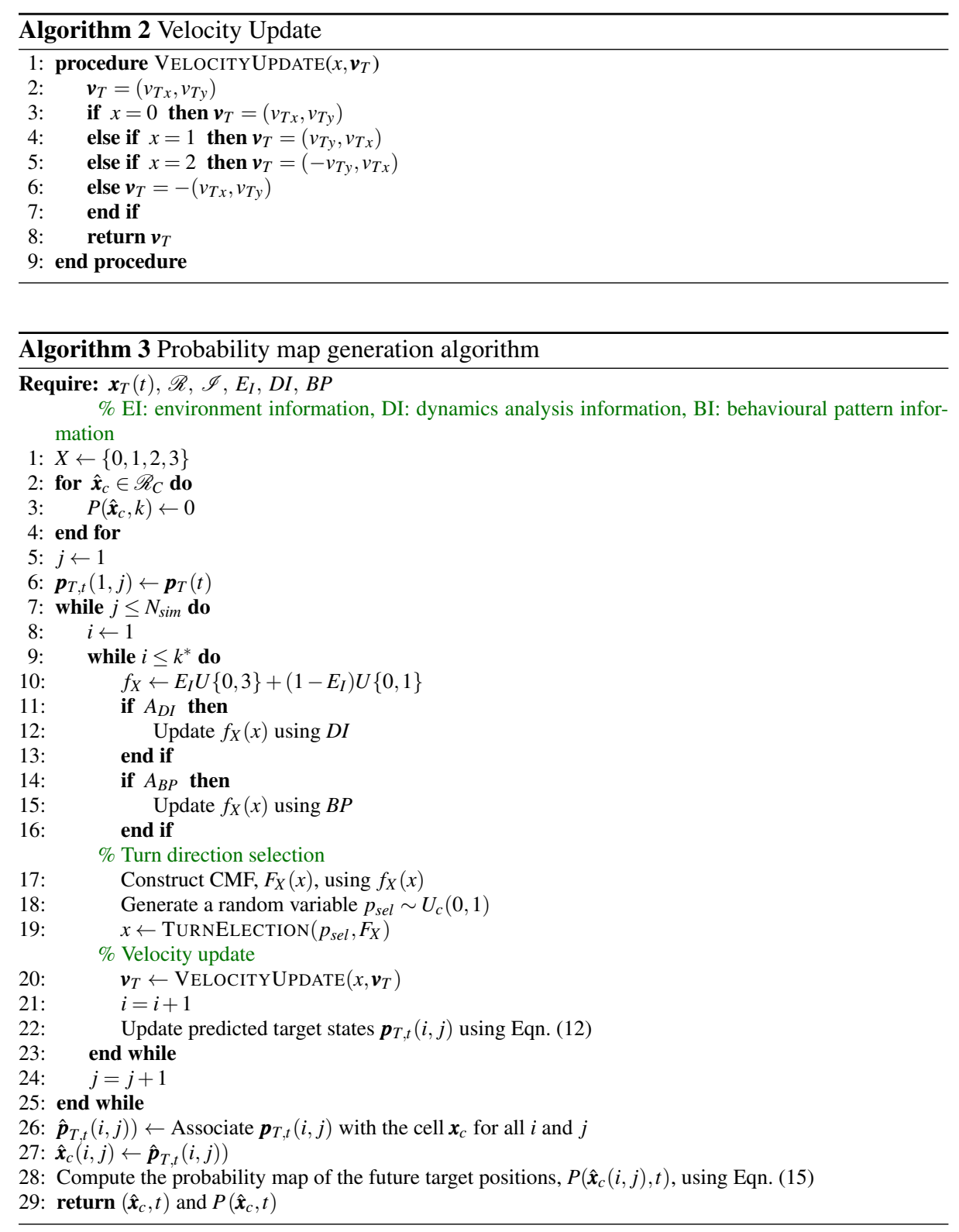

\subsubsection{Dynamics analysis information}

The analysis of the dynamics of the target can give valuable information for the prediction of the future target position. We can state that:

- If the velocity is bigger than a given value, $V_{1}$, changing direction is not feasible and, therefore, we can assume that the target will continue in the same direction. 
- If the velocity is such that $V_{2}<v_{T} \leq V_{1}$, where $V_{2}$ is another predefined velocity, we can assume that the target will be able to turn but it will not be able to complete a U-turn manoeuvre.

- If the velocity is less than or equal to $V_{2}$ then the target will be dynamically able to perform any kind of manoeuvre.

- In addition, if the velocity is less than a given velocity, $V_{3}$, or stopped we will expect that at some point it will start moving and therefore we will assume it is driving at $v_{T}=V_{3}$.

Figure 4 summarizes the aforementioned velocities.

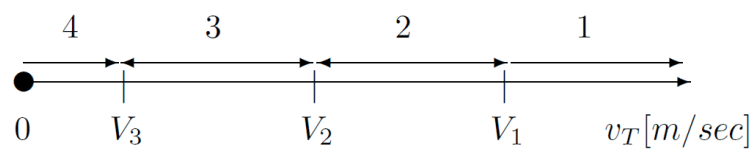

Fig. 4 Sketch with the characteristics velocities

Based on this dynamics analysis information, the PMF can be updated as:

$$
f_{X}(x)=\frac{\delta_{x 0}+\mathscr{I}_{\left[0, V_{2}\right)}\left(v_{T}\right) \delta_{x 1}+2 E_{I} \mathscr{I}_{\left[0, V_{1}\right)}\left(v_{T}\right) U\{2,3\}}{1+\mathscr{I}_{\left[0, V_{2}\right)}\left(v_{T}\right)+2 E_{I} \mathscr{I}_{\left[0, V_{1}\right)}\left(v_{T}\right)}
$$

where $\mathscr{I}_{A}(z)$ denotes the indicator function, which is defined as:

$$
\mathscr{I}_{A}(z):=\left\{\begin{array}{ll}
1, & \text { if } z \in A \\
0, & \text { if } z \notin A
\end{array},\right.
$$

and $\delta_{x a}$ represents the Kronecker delta:

$$
\delta_{x a}:= \begin{cases}1, & \text { if } x=a \\ 0, & \text { if } x \neq a\end{cases}
$$

\subsubsection{Behavioural pattern information}

As emphasised, the behavioural patterns of the target are also utilised in refining the probability distribution of the predicted future positions of the target. The leading principle is simple: if the turn direction of the target complies with the allowable directions in the behavioural pattern identified, then we should increase the probability of that direction and decrease probability of the other directions. Given the $i^{t h}$ behavioural patten identified, applying this principle, the PMF can be updated as:

$$
\bar{f}_{X}(x):=\frac{f_{X}(x)-r(x)}{\sum_{x=0}^{3} \bar{f}_{X}(x)}
$$

where $\bar{f}_{X}$ and $f_{X}$ denote the updated and previous PMFs, respectively, and $r(x)$ is a reduction factor that is defined as:

$$
r(x)=f_{X}(x) \cdot \mathscr{A}_{i}(x) \cdot K
$$


Here, $\mathscr{A}_{i}(x)$ is given by:

$$
\left\{\begin{array}{l}
\mathscr{A}_{i}(x)=0, \quad \text { if } x \text { is allowed in the behavioural pattern identified, } \\
\mathscr{A}_{i}(x)=\mathscr{A}_{i}, \quad \text { otherwise. }
\end{array}\right.
$$

where $\mathscr{A}_{i}$ denotes the accuracy which measures how well the behaviour of the target matches to the $i^{\text {th }}$ pattern. Note that a factor, $K$, with a value of 0.95 has been added in the reduction factor defined in Eqn. (31). This factor ensures that, in the case of a unit accuracy, the probability of the cells is not reduced to 0 . Then, we have an update: $\bar{f}_{X}(x) \rightarrow f_{X}(x)$. For the case where the number of the behavioural patterns is larger than one, $f_{X}(x)$ is repeatedly updated using Eqn. (30) for all $\mathscr{A}_{i}(x)$

The accuracy is the key enabler for incorporating the behavioural information into the probability refinement and it varies depending on the behavioural patterns. Therefore, how to define behavioural patterns is critical. This paper defines four behavioural patterns, and designs the accuracy depending on these patterns. Note that the behavioural recognition strategies used in this paper are simple since they are not the main focus of this study. If more sophisticated or advanced behavioural recognition is available, it can be readily incorporated into the proposed framework. Moreover, if appropriated, more behavioural patterns can be easily taken into account.

Now, let us discuss the behavioural patterns used in this paper and derivation of the accuracy for each behavioural pattern.

"Following the traffic rules" Given the scenario defined in Section 3.2 of a city with road directions, if the target is following the traffic rules, we can use it to enhance the prediction of the future target position. The accuracy, $\mathscr{A}$, of the behaviour is based on the number of times the target does not follow the traffic rules:

$$
\mathscr{A}=\left\{\begin{array}{l}
1-\frac{\text { infractions }}{\text { infractions }+2} \\
0 \text { otherwise }
\end{array} \text { if infractions } \leq \gamma \in \mathbb{N}^{0}\right.
$$

where infractions counts the number of times the target does not follow the rules and $\gamma$ is a threshold. Note that we assume that if the target does not follow the traffic rules more than the threshold we will consider as 0 the accuracy of the behaviour. The value of threshold has been set based on the results obtained in different simulations.

Figure 5 shows an example illustrating how this is translated into the probabilistic map.

"Target that follows the shortest path, (SP)" This pattern implies that the target will choose the shortest path in order to arrive to an unknown destiny. In order to analyse if the target is following the shortest path we will check at each step if the distance from the original point to the current state is increasing. Therefore, the condition of the "Shortest path" behaviour can be reduced to:

$$
\operatorname{Cond}_{S P}: \quad\left\|\boldsymbol{p}_{T}(t)-\boldsymbol{p}_{T}(0)\right\|>\left\|\boldsymbol{p}^{T}(t-1)-\boldsymbol{p}_{T}(0)\right\|
$$

It is important to note that the distances defined above are not Euclidean distances but road distances and, therefore, they are constrained by the environment. The accuracy, $\mathscr{A}$, is defined as:

$$
\left\{\begin{array}{l}
\mathscr{A}=1 \text { if } \operatorname{Cond}_{S P} \\
\mathscr{A}=0 \text { if } \neg \operatorname{Cond}_{S P} .
\end{array}\right.
$$




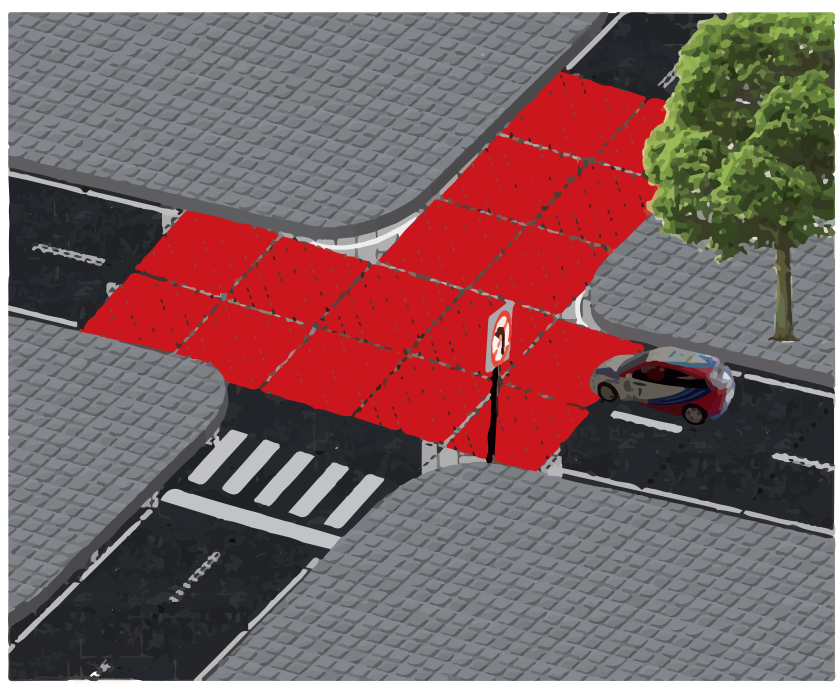

Fig. 5 Illustration of the probabilistic map for a target which is following the traffic rules. When the target reaches the intersection, given that turning left is not allowed, if the target is following the traffic rules we can enhance the probabilities of turning right and staying in the same direction.

In order to calculate the distances in an urban environment with defined directions a Dijkstra algorithm using a priority queue has been implemented. The basic algorithm is detailed in [48].

"Decelerate before turning" An important source of information can be obtained if the target decelerates before turning, given that in this way we could enhance the probability of the turning if it decelerates or the probability of continuing in the same direction otherwise. In order to correctly apply this framework the following must be fulfilled. First, the target must be traveling in the straight section at velocities higher than the maximum turning velocity. Note that, therefore, in order to perform a turning a deceleration is needed. It is also essential to check if the target decelerates when it is going to follow in the same direction. If it does not, then, we can expect that if the target decelerates it will turn.

The accuracy is defined as:

$$
\mathscr{A}=0.2 \cdot N_{1}+0.8 \cdot N_{2}
$$

where

$$
N_{1}=\frac{D_{t}}{N_{\text {Turnings }}}, \quad N_{2}=\frac{N D_{t}}{N_{\text {Forward }}}
$$

Here, $D_{t}$ denotes the number of times the target decelerated before turning and $N_{\text {Turnings }}$ the total number of turnings performed by the target. In addition, $N D_{t}$ is the number of times the target did not decelerate when approaching an intersection before deciding to keep in the same direction and $N_{\text {Forward }}$ the number of times the target continued in the same direction. As shown in Eqn. (36), higher importance is given to the term that accounts if the target does not decelerate when it is going to continue in the same direction.

"High turning ratio" If we detect that the target is performing a significant number of turnings we can use this information in order to improve the probability of target changing 
direction at the next intersection. This framework is incorporated into our proposed algorithm by considering the turning ratio $\left(T_{r}\right)$, i.e. the ratio between the number of turnings and the total number of intersections. If the turning ratio is bigger than 0.85 , this framework is triggered and its accuracy is defined as:

$$
\mathscr{A}=\frac{5}{3} T_{r}-\frac{2}{3}
$$

It is important to note that a combination of any of the previous behaviour frameworks can also be detected producing a more accurate probabilistic map.

\section{Persistent Sensing Guidance}

\subsection{Optimal Persistent Sensing Guidance Problem}

We define the optimal persistent sensing guidance problem as follows: Optimal guidance problem $1\left(\Pi_{1}\right)$ :

$$
\min _{\left[u_{1}, \ldots, u_{N_{U}}\right]} J=\min _{\left[u_{1}, \ldots, u_{N_{U}}\right]} \sum_{i=1}^{N_{U}} J_{i}\left(\mathscr{V}, \hat{\boldsymbol{p}}_{T}, \boldsymbol{p}_{i}, u_{i}, k, t^{*}\right)
$$

subject to the system dynamics described in Eqn. (10) and the constraints defined as:

$$
\begin{aligned}
& s_{i j}>d_{\min }, \quad \forall i, j \text { such that } i \neq j \\
& \left\|u_{i}\right\| \leq \frac{v_{i}^{2}}{R_{i, \min }}, \quad \forall i
\end{aligned}
$$

where $J_{i}$ denotes the objective function of the $i^{\text {th }} \mathrm{UAV}, \mathscr{V}$ is a measure of the coverage, i.e. visibility, and $R_{i, \text { min }}$ denotes the minimum turning radius of the $i^{t h} \mathrm{UAV}$.

$\hat{\boldsymbol{p}}_{T}$ represents the predicted target position vectors over the time window of $\left[t, t+t^{*}\right]$ at time step $t$ and $\boldsymbol{p}_{i}(s):=\left(x_{i}(s), y_{i}(s), z_{i}\right)$ with $z_{i}$ denoting the operational altitude of the $i^{t h}$ UAV. In addition, $\boldsymbol{s}_{i}$ denotes the vector which consists of distances from the $i^{t h}$ UAV to the other UAVs, so the $j^{\text {th }}$ component of the vector $\boldsymbol{s}_{i}$ is given by:

$$
s_{i j}=\left\|\boldsymbol{p}_{i}-\boldsymbol{p}_{j}\right\| \quad i \neq j
$$

Note that the first inequality constraint in Eqn. (40) is for collision avoidance among UAVs. Since it is assumed that the operational altitudes of UAVs are higher than those of buildings, obstacle avoidance with the buildings is not considered in the problem.

As it has been discussed in Section 2, there have been many different approaches of addressing the persistent guidance problem in the related work. One of the common features of these approaches is that they involve multiple objectives. There are mainly three approaches to multi-objective optimisation problems: a priori, a posteriori, and interactive [49]. Since the main focus of this study is to investigate the performance improvement by utilising more information in persistent sensing, we use the a priori approach, that is the weighted-sum approach. Note that a priori methods are widely used thanks to their simplicity and intuitiveness [50]. Developing more appropriate multi-objective optimisation in persistent sensing guidance will be a subject of future study.

Applying the a priori approach, the objective function for each UAV is a simple weighted aggregation of objectives:

$$
J_{i}=\sum_{n=1}^{N_{i, o b j}} W_{n} J_{i}^{n}
$$


where $N_{i, o b j}$ is the number of objectives of the $i^{\text {th }}$ UAV. For the simplicity, but without lose of generality, this paper considers two objectives which are defined identical for each UAV, so $N_{i, o b j}=2$ for $i \in\left\{1, \ldots, N_{U}\right\}$.

The first objective considered is the target coverage. As stated in Introduction, this paper aims to design UAVs paths to maximise the line-of-sight availability. This implies that the main objective of this study is to enable as much target coverage as possible. It is more important to maintain visibility to more probable target positions. Therefore, it will be beneficial to maximise not only the current coverage of the target, but also the future coverage. As maximising the visibility is equivalent to minimising the probability of losing the target, the first objective of the $i^{\text {th }} \mathrm{UAV}$ can defined as:

$$
J_{i}^{i}=\int_{t}^{t+t^{*}} \mathscr{V}^{c}\left(\hat{\boldsymbol{p}}_{T}, \boldsymbol{p}_{i}(s), \mathscr{E}\right) d s
$$

where $\boldsymbol{p}_{i}(s):=\left(x_{i}(s), y_{i}(s), z_{i}\right)$ with $z_{i}$ denoting the operational altitude of the $i^{\text {th }}$ UAV. In addition, $\mathscr{V}^{c}(\cdot, \cdot, \cdot)$ is the complement of the coverage, i.e. the function of losing the target, which is defined as:

$$
\mathscr{V}^{c}\left(\hat{\boldsymbol{p}}_{T}, \boldsymbol{p}_{i}(s), \mathscr{E}\right)=\int_{R_{f t p}}\left\{1-\mathscr{V}\left(\hat{\boldsymbol{p}}_{T}, \boldsymbol{p}_{i}(s), \mathscr{E}\right)\right\} f\left(\hat{\boldsymbol{p}}_{T}\right) d R_{f t p}
$$

where $R_{f t p}$ is the target candidate region and $f\left(\hat{\boldsymbol{p}}_{T}\right)$ denotes the probability density function of the predicted target position vectors $\hat{\boldsymbol{p}}_{T}$. If the LOS is not occluded by any building and the target is within the maximum sensing range, the target is assumed to be visible. Therefore, the visibility is given as:

$$
\left\{\begin{array}{l}
\mathscr{V}=1, \text { if } \boldsymbol{r} \cap \mathscr{E}=\varnothing \&|\boldsymbol{r}|<R_{C} \\
\mathscr{V}=0, \text { otherwise. }
\end{array}\right.
$$

where $R_{C}$ and $\boldsymbol{r}$ denote the maximum sensing range and the relative position vector of the UAV with respect to the target, respectively.

Because it is generally desirable to accomplish the mission with the minimum control cost, the second objective function considered is the total control effort over the given time window. Hence, the second objective function for the $i_{t h}$ UAV is defined as:

$$
J_{i}^{2}=\frac{1}{2} \int_{t}^{t+t^{*}} u_{i}^{2}(s) d s
$$

Remark 1 Because the objective function described by Eqns. (43) and (44) is in general non-convex, the optimal guidance problem $\Pi_{1}$ is generally not a convex program. Moreover, the feasibility set defined by the collision avoidance constraint in Eqn. (40) is non-convex. As the operational environment becomes more complicated, e.g. density of the building and number of UAVs increase, $\Pi_{1}$ becomes more complicated to be solved. This implies that finding the exact solution of $\Pi_{1}$ in a polynomial time is not likely feasible.

\subsection{Approximated Optimal Guidance Problem}

In order to mitigate the computation issue resulting from the non-convexity, this paper approximates the optimal guidance problem $\Pi_{1}$. By the approximation, it is expected that the optimal solution of the approximated problem can be obtained in a matter of a few seconds. The approximated optimal guidance problem is formulated as follows. 
The optimal guidance problem $2\left(\Pi_{2}\right)$ :

$$
\min _{\left[\dot{\psi}_{1}, \ldots, \dot{\psi}_{N_{U}}\right]} J=\min _{\left[\dot{\psi}_{1}, \ldots, \dot{\psi}_{N_{U}}\right]} \sum_{i=1}^{N_{U}} J_{i}\left(\mathscr{V}, \hat{\boldsymbol{p}}_{T}, \boldsymbol{p}_{i}, \psi_{i}, k, t^{*}\right)
$$

subject to the system dynamics described in Eqn. (10) and the constraints defined as:

$$
\left\|\dot{\psi}_{i}\right\| \leq \dot{\psi}_{\max }, \quad \forall i
$$

where

$$
\dot{\psi}_{i} \in \dot{\psi}_{\max } \cdot\left\{-1,-\frac{3}{4},-\frac{1}{2},-\frac{1}{4}, 0, \frac{1}{4}, \frac{1}{2}, \frac{3}{4}, 1\right\}
$$

As can be noticed, $\Pi_{2}$ is discretised to reduce computational load in finding the solution of the optimal guidance problem. Since it is assumed that each UAV maintains a constant speed, $\Pi_{2}$ is to find the heading angle rate commands from a set of discretised turning ratios. Note that other well-known algorithms such as the algorithm proposed by Cook et al. [46] also accepted this approximation to relax computation burden. The control input can be simply retrieved from the relation between the heading angle rate and the lateral acceleration, given in the form:

$$
u_{i}=v_{i} \dot{\psi}_{i}
$$

Remark 2 The solution of the approximated optimal guidance problem provides an approximated solution of the original optimal guidance problem $\Pi_{1}$. Ideally, if the feasible set defined by Eqn. (40) is discretised and an exhaustive search is performed, the optimal solution can be found [51]. Therefore, as the number of the set of discretised inputs in Eqn. (49), the approximated solution of $\Pi_{2}$ is expected to become closer to the optimal solution of $\Pi_{1}$ at the expense of increased computational load.

The first objective function for each UAV is also discretised as:

$$
\begin{aligned}
J_{i}^{1} & =\sum_{m=1}^{k^{*}} \sum_{n=1}^{N_{s i m}}\left(1-\mathscr{V}\left(\hat{\boldsymbol{p}}_{T, t}(m, n), \boldsymbol{p}_{i}(t+m \cdot \Delta t), \mathscr{E}\right)\right) \cdot P\left(\hat{\boldsymbol{p}}_{T, t}(m, n)\right) \\
& =\sum_{m=1}^{k^{*}} \sum_{n=1}^{N_{p}}\left(1-\mathscr{V}\left(\hat{\boldsymbol{x}}_{c}(m, n), \boldsymbol{p}_{i}(t+m \cdot \Delta t), \mathscr{E}\right)\right) \cdot P\left(\hat{\boldsymbol{x}}_{c}(m, n), t\right)
\end{aligned}
$$

where $N_{p}$ is the number of associated cell with the predicted target positions. The second objective function is then discretised as:

$$
J_{i}^{2}=\frac{1}{2} \sum_{m=1}^{k^{*}} u_{i}^{2}(t+(m-1) \cdot \Delta t)
$$

Note that the collision avoidance constraint is excluded in $\Pi_{2}$. Since the collision avoidance constraint is not convex, considering this as a hard constraint could result in high computational burden. Alternatively, the collision avoidance constraint can be considered as a soft constraint. Therefore, for collision avoidance, an additional objective function is introduced in the proposed approximated optimal guidance problem:

$$
J_{i}^{3}=\sum_{m=1}^{k^{*}}\left(\sum_{j=1}^{j=N_{U A V s}} s_{i j}(t+m \cdot \Delta t) \cdot f\left(s_{i j}(t+m \cdot \Delta t), d_{m i n}, \Delta d\right)\right)
$$


where

$$
f\left(d, d_{0}, \Delta d\right)=\frac{1}{2}\left[1-\tanh \left(\frac{d-d_{0}-\frac{\Delta d}{2}}{\frac{\Delta d}{8}}\right)\right]
$$

Note that $f(\cdot, \cdot, \cdot)$ is a hyperbolic tangent function that was also utilised for collision avoidance in similar problems [30]. Consequently, the number of objective functions for each UAV is increased by one, compared to that in $\Pi_{1}$.

\section{Empirical Validation}

In this section numerical simulations are performed to assess the performance of the proposed persistent sensing guidance. The prime purpose of the empirical validation is to investigate whether the proposed framework actually improves the performance of the persistent sensing when additional available information of the target is utilised. Therefore, this paper leaves out any comparison with the existing persistent sensing guidance algorithm. Instead, the performance of the proposed approach with different level of information is examined and compared with each other.

The mission environment utilised in the simulations is a Manhattan-type urban environment of $10 \times 10 \mathrm{~m}^{2}$, which is shown in Figure 1. Note that as proof of the concept and feasibility, this type of mission environment is used. However, the proposed framework is not constrained to be applied only to such a mission environment; more complicated and complex environments can be readily incorporated into the proposed framework.

For the rigorous validation, we performed Monte Carlo simulations with 100 independent scenarios. The scenarios have been divided into 5 different sets aiming at assessing the four behavioural patterns discussed in this paper and an additional set where the target is randomly manoeuvring. Note that the last set is considered to examine any performance degradation in the case where there is no behavioural pattern identified by the proposed framework. Each set contains scenarios for which the target meets a specific behavioural pattern. In all the scenarios two UAVs are tracking a single non-cooperative ground target that meets the dynamic constraints defined in the target's dynamic definition.

The numerical parameters of the different simulations have been summarised in table 1. In addition, Figure 6 shows a sample trajectory of a target that follows the traffic rules. A realistic number of one-way roads has been considered. Note that in a scenario with no one-way road, this behaviour would not provide any information. For each scenario, we carried out four different simulations in which different level of target information is taken into account. In the first simulation, no target information is used by the persistent sensing guidance algorithm and, therefore the same probability is assigned in the target's prediction. In the second execution, only dynamic information is exploited. In the third execution, the road information is used and, finally in the last execution all the target information is used. In this way, the relative enhancement introduced by the different information sources can be identified.

In order to assess the maximum potential benefits of the behaviour identification, an initial behavioural record, with the appropriate information for each pattern, is assumed for the target. In this way, the initial pattern recognition time is highly reduced and the assessment can be performed from the starting point. This prevents from requiring enormous testing scenarios that would highly increase the computational load.

The simulation results are summarised in Table 2 and depicted in Figure 7. The visibility ratio, defined as the number of time stamps where at least one of the UAVs tracks the target, 


\begin{tabular}{|c|c|}
\hline \multicolumn{2}{|c|}{ Values of the parameters } \\
\hline Target's characteristic velocities & $V_{1}=22 \mathrm{~m} / \mathrm{s}, V_{2}=18 \mathrm{~m} / \mathrm{s}, V_{3}=4 \mathrm{~m} / \mathrm{s}$ \\
UAVs constant velocity & $29 \mathrm{~m} / \mathrm{s}$ \\
Prediction horizon, $t^{*}$ & $t^{*}=7$ time steps \\
Minimum turning radius & $100 \mathrm{~m}$ \\
UAVs altitude & $100 \mathrm{~m}$ \\
\hline height, Buildings density, $\rho_{b}$ & $50 \mathrm{~m}, 60 \%$ \\
Road width, $R_{W}$ and Occupancy factor, $F_{b}$ & $20 \mathrm{~m}$ and $80 \%$ \\
Cell size & $10 \mathrm{~m}$ \\
\hline Time step, $\Delta T$ & $1 \mathrm{sec}$ \\
Number of UAVs & 2 \\
Number of targets & 1 \\
\hline
\end{tabular}

Table 1 Values of the parameters for the execution of the simulations for a driver that follows the traffic rules.

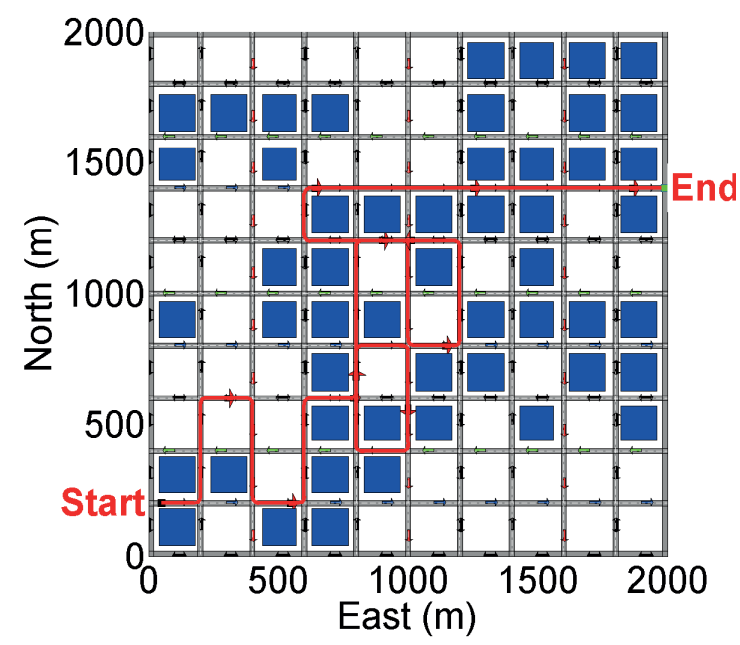

Fig. 6 Sample trajectory of a target that follows traffic rules.

is used in the performance assessment. The percentage of the performance improvement, denoted as $\Delta$ in Figure 7, is obtained as:

$$
\Delta=\frac{\mathscr{V}_{a, \text { info }}-\mathscr{V}_{a, \text { no }}}{\mathscr{V}_{a, \text { no }}} \times 100
$$

where $\mathscr{V}_{a \text {,info }}$ and $\mathscr{V}_{a \text {,no }}$ are average visibility with available information and without any information. In case where the target is randomly manoeuvring, as expected, it is evident that the dynamic information provides the most valuable information given that the dynamic constraints are met. Regarding the targets following a given pattern, it can be seen in Table 2 that incorporating only the dynamic information to the proposed framework improves the visibility conditions by around $14 \%$, including the results from a random target. A similar enhancement is introduced from the use of road information. Utilising all available information of the target improves the visibility by more than $19 \%$, taking the results of a random target into account, and the enhancement becomes significant especially when a certain behavioural pattern that the target follows is identified. All the simulation results confirm that the proposed innovation of this paper: leveraging the available information 
of the target under the proposed framework dramatically improves the performance of the persistent sensing.

\begin{tabular}{|l|l|l|l|l|}
\hline Target's behaviour & $\begin{array}{l}\text { No informa- } \\
\text { tion }\end{array}$ & $\begin{array}{l}\text { Dynamic } \\
\text { information }\end{array}$ & $\begin{array}{l}\text { Road infor- } \\
\text { mation }\end{array}$ & $\begin{array}{l}\text { All informa- } \\
\text { tion }\end{array}$ \\
\hline Random behaviour & 0.7 & 0.75 & 0.71 & 0.75 \\
\hline $\begin{array}{l}\text { Target that follows traffic rules } \\
\text { (TFTR) }\end{array}$ & 0.71 & 0.84 & 0.86 & 0.90 \\
\hline Shortest path target (SPT) & 0.68 & 0.81 & 0.83 & 0.89 \\
\hline $\begin{array}{l}\text { High Turning Ratio Target } \\
\text { (HTRT) }\end{array}$ & 0.7 & 0.78 & 0.79 & 0.85 \\
\hline Decelerating target (DT) & 0.65 & 0.74 & 0.72 & 0.81 \\
\hline
\end{tabular}

Table 2 Average visibility conditions.

It is important to note that, the performances obtained in the scenarios where no target information is used also slightly depend on the target's behaviour pattern identified. This is due to the fact that the different patterns lead to trajectories of different characteristics, i.e. even a random target following the traffic rules and a High turning ratio target for a certain period of time could exhibit trajectories with a higher percentage of going around a building meanwhile the remaining behaviours lead to more straight trajectories. When identified, this information could help in enhancing the performance of our proposed approach.

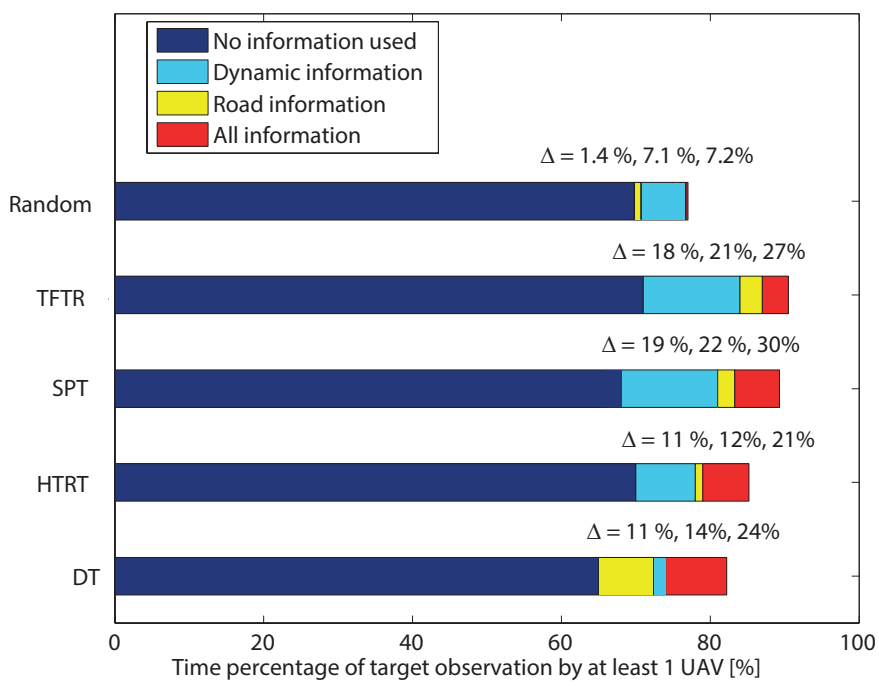

Fig. 7 Comparative results of the different behaviour frameworks defined. The accumulated relative enhancement with respect to the no information case has been included in the different cases.

As discussed in Section 2, there have been some existing results reported. A persistent sensing algorithm, which is proposed by Wang and Cao [33], achieved a constant sensing an average $95 \%$ of the time. This result are slightly better than the results obtained by the 
framework proposed in this paper, but the algorithm in [33] used 3 UAVs, not 2 UAVs, and are simulated in an urban environment with a reduced building density. The work from Yu et al. [45] obtained an $85 \%$ of visibility by using a single UAV and unmanned ground vehicle (UGV) with a target following a simple rectangular trajectory. Note that the results in $[33,45]$ are not directly comparable to those of the proposed approach as the simulation conditions are different.

As discussed in Section 5.2, the algorithm proposed by Cook et al. [46] are developed in a similar optimal control framework. Moreover, their simulation conditions are similar to the conditions assessed in this paper. The algorithm by Cook et al. [46] obtained a continuous visibility of around $70 \%$ of the time for probabilistic targets, which is similar to the average performance of our algorithm in the case where no information is utilised. When more information are incorporated in the framework developed in this paper, the incremental performance improvement over the exiting results is evident. This confirms that the proposed approach represents an added value to previous similar approaches.

An assessment of the applicability of the proposed approach in real scenarios is also justified. For the assessment, the computational load of the algorithm is assessed. The scenarios are executed in MATLAB(2013b) on a standard specification PC, an Intel(R) Core(TM) i5$5300 \mathrm{CPU}$ operating at $2.30 \mathrm{GHz}$. The processing time of the scenarios is recorded and the average processing time per time stamp, computed as the time required for the completion of the scenario divided by the number of time stamps of the scenario, is investigated. The average processing time per time stamp is $0.8 \mathrm{~s}$. Note that the simulation environment is build on MATLAB. The assessment result indicates real-time applications of the proposed approach are feasible with code and time optimization.

The extrapolation of the simulation results to a practical scenario is sensitive to highly unstable communications between the UAVs which would not allow an optimal cooperative tracking and to sensing uncertainties which would impact the performance of the proposed information-driven algorithm. However, the formulation of this work, designed as a modular algorithm that can be added to any existing system, ensures an added value even in harsh environments.

\section{Conclusion}

The persistent monitoring of non-cooperative ground targets in urban environments with a swarm of UAVs was studied. The UAVs were assumed to be point masses with dynamic constraints (i.e., Dubins vehicles) and a 2D space problem is considered. Control system is assumed as well designed and its bandwidth is separated from the guidance loop. Wind disturbances are out of the scope of this work. The algorithm proposed takes into account the occlusion problems due to the buildings, which are modeled as polyhedrons, and aims at designing trajectories to maximise the current and future visibility of the target. A probabilistic approach is used in order to guess the future target position. The key innovation introduced in this work is to take advantage of available target information in order to enhance the computation of the probability distribution of the future target position. The proposed algorithm is able to identify a set of predefined behavioural patterns and to take the target's dynamics that can be used to refine the probability distribution of the predicted future target positions. For the path planning an a priori approach is used in order to solve the multi-objective optimization problem. In order to ease the computational burden the optimal guidance problem has been discretised. The optimal solution comes from the minimization 
of a simple weighted aggregation of two objectives, the target visibility and the control and with a constraint in order to account for the collision avoidance.

The algorithm has been tested in a simulated environment consisting in a Manhattantype urban map whose buildings are polyhedrons. Monte Carlo simulations have been executed. The results show that the visibility performance of the persistent monitoring problem are increased around $14 \%$ if the dynamic information is used and up to $30 \%$ if one of the behavioural patterns is identified, which represents an added value when compared to similar related works.

The proposed framework has proven the feasibility of the application of target's dynamic constraints and behavioural recognition to the persistent sensing problems. Further refinement and elaboration introducing even more realistic environments are justified. Moreover, the modularity of the algorithm easily allows the definition of more behavioural patterns that can be identified as potentially beneficial.

\section{References}

1. D. Kingston, R.W. Beard, and R.S. Holt. Decentralized perimeter surveillance using a team of UAVs. IEEE Transactions on Robotics, 24(6):1394-1404, 2008.

2. D. Bein, W. Bein, A. Karki, and B.B. Madan. Optimizing border patrol operations using unmanned aerial vehicles. pages 479-484, 2015.

3. S. Minaeian, J. Liu, and Y.-J. Son. Vision-based target detection and localization via a team of cooperative uav and ugvs. IEEE Transactions on Systems, Man, and Cybernetics: Systems, 46(7):1005-1016, 2016.

4. R.R. Pitre, X.R. Li, and R. Delbalzo. UAV route planning for joint search and track mission - an informative-value approach. IEEE Transactions on Aerospace and Electronic Systems, 48(3):25512565, 2012

5. B.H. Lim, J.W. Kim, S.W. Ha, and Y.H. Moon. Development of software platform for monitoring of multiple small uavs. volume 2016-December, 2016.

6. P. Li and H. Duan. A potential game approach to multiple uav cooperative search and surveillance. Aerospace Science and Technology, 68:403-415, 2017

7. Anuj Puri. A survey of unmanned aerial vehicles (UAV) for traffic surveillance. Technical report, Department of Computer Science and Engineering, University of South Florida, 2004.

8. E.M. Carapezza and D.B. Law. Sensors, c3i, information, and training technologies for law enforcement. In Proc. SPIE, January 1999.

9. A. Belbachir and J.-A. Escareno. Autonomous decisional high-level planning for uavs-based forest-fire localization. volume 1, pages 153-159, 2016

10. Eva Ericsson. Driving pattern in urban areas - descriptive analysis and initial prediction model. Technical report, Lunds universitet, instutionen fr teknik och samhlle, trafik och vg, 2000.

11. Firas Lethaus, Martin R. K. Baumann, Frank Köster, and Karsten Lemmer. Using Pattern Recognition to Predict Driver Intent, pages 140-149. Springer Berlin Heidelberg, Berlin, Heidelberg, 2011.

12. Mohd Nizam Husen, Sukhan Lee, and Muhammad Qasim Khan. Syntactic pattern recognition of car driving behavior detection. In Proceedings of the 11th International Conference on Ubiquitous Information Management and Communication, IMCOM '17, pages 77:1-77:6, New York, NY, USA, 2017. ACM.

13. J. Kim and Y. Kim. Moving ground target tracking in dense obstacle areas using UAVs. In IFAC World Congress, COEX, South Korea, 2008.

14. H. Chen. UAV path planning with tangent-plus-lyapunov vector field guidance and obstacle avoidance. IEEE Transactions on Aerospace and Electronic Systems, 49(2):840-856, 2013.

15. S. Lim, Y. Kim, D. Lee, and H. Bang. Standoff target tracking using a vector field for multiple unmanned aircrafts. Journal of Intelligent and Robotic System, 69(1-4):347-360, 2013.

16. W. Jung, S. Lim, D. Lee, and H. Bang. Unmanned aircraft vector field path following with arrival angle control. Journal of Intelligent and Robotic Systems: Theory and Applications, 84(1-4):311-325, 2016.

17. W. Jiang, D. Wang, Y. Wang, and Z.A. Ali. Uav rendezvous based on time-varying vector fields. Electronics Letters, 53(10):653-655, 2017.

18. I. Shames, S. Dasgupta, B. Fidan, and B.D.O. Anderson. Circumnavigation using distance measurements under slow drift. IEEE Transactions on Automatic Control, 57(4):889-903, 2012. 
19. M. Deghat, I. Shames, B.D.O. Anderson, and C. Yu. Target localization and circumnavigation using bearing measurements in 2D. In IEEE Conference on Decision and Control, 2010.

20. R.A. Livermore. Optimal UAV path planning for tracking a moving ground vehicle with a gimbaled camera. Master's thesis, US Air Force Institute of Technology (USAF), 2014.

21. Fahd Rafi, Saad Khan, Khurram Shafiq, and Mubarak Shah. Autonomous target following by unmanned aerial vehicles. In Defense and Security Symposium, pages 623010-623010. International Society for Optics and Photonics, 2006.

22. R.A. Wise and R.T. Rysdyk. UAV coordination for autonomous target tracking. AIAA Guidance, Navigation and Control Conference, Keystone, Colorado, USA, 2006.

23. S. Yoon, S. Park, and Y. Kim. Circular motion guidance law for coordinated standoff tracking of a moving target. IEEE Transactions on Aerospace and Electronic Systems, 49(4):2440-2462, 2013.

24. S. Kim, H. Oh, and A. Tsourdos. Nonlinear model predictive coordinated standoff tracking of moving ground vehicle. Journal of Guidance, Control and Dynamics, 36(2):557-566, 2013.

25. D. Kingston and R. Beard. UAV spaly state configuration for moving targets in wind. Lecture Notes in Control and Information, 369:109-128, 2007.

26. T.H. Summers, M.R. Akella, and M.J. Mears. Coordinated standoff tracking of moving targets: Control laws and information architectures. Journal of Guidance, Control, and Dynamics, 32(1):56-69, 2009.

27. E.W. Frew. Sensitivity of cooperative target geolocalization to orbit coordination. Journal of Guidance, Control, and Dynamics, 31(4):1028-1040, 2008.

28. H. Oh, S. Kim, A. Tsourdos, and B.A. White. Road-map assisted standoff tracking of moving ground vehicle using nonlinear model predictive control. In American Control Conference, Montreal, Canada, June 2012.

29. C. Zhao, M. Zhu, H. Liang, and Z. Wu. The sustainable tracking strategy of moving target by uav in an uncertain environment. volume 2016-August, pages 5641-5647, 2016.

30. Zhirong He and Jian-Xin Xu. Moving target tracking by uavs in an urban area. In Control and Automation (ICCA), 2013 10th IEEE International Conference on, pages 1933-1938. IEEE, 2013.

31. P. Yao, H. Wang, and Z. Su. Real-time path planning of unmanned aerial vehicle for target tracking and obstacle avoidance in complex dynamic environment. Aerospace Science and Technology, 47:269-279, 2015.

32. Mingfeng Zhang and Hugh HT Liu. Persistent tracking using unmanned aerial vehicle: A game theory method. In AIAA Guidance, Navigation, and Control Conference, pages 8-11, 2011.

33. Y. Wang and Y. Cao. Coordinated target tracking via a hybrid optimization approach. Sensors (Switzerland), 17(3), 2017.

34. Zhijun Tang and Umit Ozguner. Motion planning for multitarget surveillance with mobile sensor agents. IEEE Transactions on Robotics, 21(5):898-908, 2005.

35. Wang Xun, Kong Wei-Wei, Zhang Dai-Bing, and Zhu Hua-Yong. Segment guidance and control on noncooperative ground target tracking for unmanned aerial vehicles. In Control Conference (CCC), 2012 31st Chinese, pages 4868-4872. IEEE, 2012.

36. M. Ramasamy and D. Ghose. A heuristic learning algorithm for preferential area surveillance by unmanned aerial vehicles. Journal of Intelligent and Robotic Systems: Theory and Applications, pages 1-27, 2017.

37. J. Redding, A. Geramifard, A. Undurti, H.-L. Choi, and J.P. How. An intelligent cooperative control architecture. pages 57-62, 2010. cited By 13.

38. Ben Grocholsky, James Keller, Vijay Kumar, and George Pappas. Cooperative air and ground surveillance. Robotics \&amp; Automation Magazine, IEEE, 13(3):16-25, 2006.

39. Vitaly Shaferman and Tal Shima. Unmanned aerial vehicles cooperative tracking of moving ground target in urban environments. Journal of guidance, control, and dynamics, 31(5):1360-1371, 2008.

40. K. Zhang, X. Gao, and D. Chen. Three dimensional trajectory tracking for unmanned aerial vehicles in time-varying winds. pages 735-739, 2016.

41. Zhijun Tang and Ümit Özgüner. Sensor fusion for target track maintenance with multiple uavs based on bayesian filtering method and hospitability map. In Decision and Control, 2003. Proceedings. 42nd IEEE Conference on, volume 1, pages 19-24. IEEE, 2003.

42. Jesus Capitan, Luis Merino, and Anibal Ollero. Decentralized cooperation of multiple uas for multitarget surveillance under uncertainties. In Unmanned Aircraft Systems (ICUAS), 2014 International Conference on, pages 1196-1202. IEEE, 2014.

43. J. Capitan, L. Merino, and A. Ollero. Cooperative decision-making under uncertainties for multi-target surveillance with multiples uavs. Journal of Intelligent \& Robotic Systems, 84(1):371-386, 2016.

44. Manickam Ramasamy and Debasish Ghose. A heuristic learning algorithm for preferential area surveillance by unmanned aerial vehicles. Journal of Intelligent \& Robotic Systems, pages 1-27, 2017.

45. Huili Yu, Randal W Beard, Matthew Argyle, and Caleb Chamberlain. Probabilistic path planning for cooperative target tracking using aerial and ground vehicles. In American Control Conference (ACC), 2011, pages 4673-4678. IEEE, 2011. 
46. Kevin Cook, Everett Bryan, Huili Yu, He Bai, Kevin Seppi, and Randal Beard. Intelligent cooperative control for urban tracking. Journal of Intelligent \&amp; Robotic Systems, 74(1-2):251-267, 2014.

47. Frederic Bourgault, Tomonari Furukawa, and Hugh E Durrant-Whyte. Coordinated decentralized search for a lost target in a bayesian world. In Intelligent Robots and Systems, 2003.(IROS 2003). Proceedings. 2003 IEEE/RSJ International Conference on, volume 1, pages 48-53. IEEE, 2003.

48. Edsger W Dijkstra. A note on two problems in connexion with graphs. Numerische mathematik, 1(1):269-271, 1959

49. Ching-Lai Hwang and Abu Syed Md. Masud. Multiple Objective Decision Making - Methods and Applications. Springer Berlin Heidelberg, 1979.

50. R. T. Marler and J. S. Arora. Survey of multi-objective optimization methods for engineering. Structural and Multidisciplinary Optimization, 26(6):369-395, 2004.

51. Ke Zhou and Stergios I Roumeliotis. Multirobot active target tracking with combinations of relative observations. IEEE Transactions on Robotics, 27(4):678-695, 2011. 
2017-10-13

\section{Information-driven persistent sensing of a non-cooperative mobile target using UAVs}

Shin, Hyo-Sang

Springer

Shin H-S, Garcia AJ, Alvarez S, Information-driven persistent sensing of a non-cooperative

mobile target using UAVs, Journal of Intelligent and Robotic Systems, Volume 92, Issue 3-4, pp.629-643

http://dx.doi.org/10.1007/s10846-017-0719-y

Downloaded from Cranfield Library Services E-Repository 